\title{
USING QUASI-SUBORDINATION PRINCIPLE TO ESTABLISH THE COEFFICIENT ESTIMATES OF A SAKAGUCHI TYPE CLASS
}

\author{
OLUBUNMI A. FADIPE-JOSEPH*, O. D. OLAWUMI \\ Department of Mathematics, University of Ilorin, P.M.B. 1515, Ilorin, Nigeria \\ *Corresponding author: famelov@unilorin.edu.ng, famelov@gmail.com
}

Received Feb. 24, 2021

\begin{abstract}
Aвstract. The initial coefficient bounds for a class $M_{q}^{\lambda}(\gamma, t, b)$ of an analytic function involving modified q-sigmoid associated with quasi-subordination were obtained. The Fekete-Szeg $\ddot{o}$ functional and Hankel determinant for the class were investigated.

2010 Mathematics Subject Classification: 30C45.

Key words and phrases. analytic functions; univalent function; quasi-subordination; modified q-sigmoid functions; Fekete-Szegö functional.
\end{abstract}

\section{InTRODUCTION}

Let A represent the class of functions of the form:

$$
f(z)=z+\sum_{k=2}^{\infty} a_{k} z^{k},(z \in \mathbb{U})
$$

which are analytic in the open unit disc $\mathbb{U}=\{z \in \mathbb{C}:|z|<1\}$ normalised by $f(0)=0$ and $f^{\prime}(0)=1$. Also $S$ represent the class of analytic univalent and normalised function in $\mathbb{U}$.

The function $l$ is said to be subordinate to $L$, written $l(z) \prec L(z)$, if there exists a function $\omega$ analytic in $\mathbb{U}$, with $\omega(0)=0$ and $|\omega(z)|<1$ and such that $l(z)=L(\omega(z))$.

Let $\varphi(z) \leq 1(z \in \mathbb{U})$ be an analytic function such that $\frac{l(z)}{\varphi(z)}$ is analytic in $\mathbb{U}$ and

$$
\frac{l(z)}{\varphi(z)} \prec L(z)(z \in \mathbb{U})
$$

which means there exist a Schwartz function $w(z)$ such that $l(z)=\varphi(z) \cdot L(w(z)), z \in \mathbb{U}$ then $l(z) \prec_{q} L(z)$ implies $l$ is said to be quasi-subordinate to $L \in \mathbb{U}$.

DOI: $10.28924 / \mathrm{APJM} / 9-4$ 
Hence, the normal subordination $\prec$ can be gotten from the quasi-subordination $\prec_{q}$ if $\varphi(z)=$ $1,(z \in \mathbb{U})$.

Frasin [6] introduced and studied coefficient inequalities for certain classes of Sakaguchi type functions $f \in A$ when $s, b \in \mathbb{C}$ with $s \neq b$ and for some $\alpha,(0 \leq \alpha<1)$ which satisfies

$$
\operatorname{Re}\left\{\frac{(s-b) z f^{\prime}(z)}{f(s z)-f(b z)}\right\}>\alpha, z \in \mathbb{U}
$$

Recently, Olatunji [7] introduced a class

$$
\left[f^{\prime}(z)\left(\frac{(s-b) z}{f(s z)-f(b z)}\right)^{\lambda}-1\right] \prec_{q}[\Phi(z)-1], z \in \mathbb{U}
$$

when $s, b \in \mathbb{C}$ with $s \neq b$ and $\Phi(z)=1+\frac{z}{2}-\frac{z^{3}}{24}+\frac{z^{4}}{240}-\frac{z^{6}}{64}+\frac{779 z^{7}}{20160}-\ldots$, the series form of modified sigmoid function.

$\mathrm{P}$ is also denoted as the class of functions $p(z)$ analytic in $\mathbb{U}$ such that $p(0)=1$ and $\operatorname{Re}(p(z))>0$. For the purpose of our results, the following lemmas and definitions are employed.

Lemma 1.1. [2] If $\omega(z)=b_{1} z+b_{2} z^{2}+\ldots, b_{1} \neq 0$ is analytic and satisfy $|\omega(z)|<1$ in the unit disk $\mathbb{U}$, then for each $0<r<1,\left|\omega^{\prime}(z)\right|<1$ and $\left|\omega\left(r e^{i \theta}\right)\right|<1$ unless $\omega(z)=e^{i \theta} z$ for some real number $\sigma$.

Lemma 1.2. [3] Let $\omega \in \Omega=\{\omega \in A:|\omega(z)| \leq|z|, z \in \mathbb{U}\}$

If $\omega \in \Omega, \omega(z)=\sum_{n=1}^{\infty} c_{n} z^{n}(z \in \mathbb{U})$, then

$$
\left|c_{n}\right| \leq 1 n=1,2, \ldots\left|c_{2}\right| \leq 1-\left|c_{1}\right|^{2}
$$

and

$$
\left|c_{2}-\mu c_{1}^{2}\right| \leq \max \{1,|\mu|\}(\mu \in \mathbb{C})
$$

The result is sharp. The functions

$$
\omega(z)=z, \omega_{a}(z)=z \frac{z+a}{1+\bar{a} z}(z \in \mathbb{U},|a|<1) .
$$

are extremal functions.

Lemma 1.3. [5] Let $h(z)$ be a sigmoid function and

$$
G(z)=2 h(z)=1+\sum_{m=1}^{\infty} \frac{(-1)^{m}}{2^{m}}\left[\sum_{n=1}^{\infty} \frac{(-1)^{n}}{[n] !} z^{n}\right]^{m}
$$

then $G(z) \in P$, where $G(z)$ is the modified sigmoid function. 
Definition 1.1. [1] A q-analogue of the ordinary exponential function $e^{z}=\sum_{k=0}^{\infty} \frac{z^{k}}{k !}$ is defined as

$$
e_{q}^{z}=\sum_{k=0}^{\infty} \frac{z^{k}}{[k] q !}
$$

Definition 1.2. [1] A q-Sigmoid function is defined as

$$
G_{q}(z)=\frac{1}{1+e_{q}^{-z}}
$$

Definition 1.3. [4] A modified q-sigmoid is defined as

$$
\gamma_{q, m, n}(z)=\frac{2}{1+e_{q}^{-z}}=1+\left(\sum_{k=1}^{\infty} \frac{(-1)^{k}}{2^{k}}\left[\sum_{n=1}^{\infty} \frac{(-1)^{n}}{[n]_{q} !} z^{n}\right]^{k}\right)
$$

Definition 1.4. Let $\gamma(z) \in P$ be univalent, for $t \neq b, t, b \in \mathbb{C}$ and $\lambda \geq 0$, a function $f \in A$ is said to be in the class $M_{q}^{\lambda}(\gamma, t, b)$ if

$$
\left[f^{\prime}(z)\left(\frac{(t-b) z}{f(t z)-f(b z)}\right)^{\lambda}-1\right] \prec_{q}\left[\gamma_{q, m, n}(z)-1\right],(z \in \mathbb{U})
$$

for the powers take principal values only.

If there exist an analytic function $\varphi(z)$ with $|\varphi(z)| \leq 1,(z \in \mathbb{U})$, then it follows that $f \in M_{q}^{\lambda}(\gamma, t, b)$ as defined in (7).

$$
\frac{f^{\prime}(z)\left(\frac{(t-b) z}{f(t z)-f(b z)}\right)^{\lambda}-1}{\varphi(z)} \prec \gamma_{q, m, n}(z)-1,(z \in \mathbb{U})
$$

If $|\varphi(z)|=1,(z \in \mathbb{U})$ in $(8)$, then

$$
f^{\prime}(z)\left(\frac{(t-b) z}{f(t z)-f(b z)}\right)^{\lambda} \prec \gamma_{q, m, n}(z),(z \in \mathbb{U})
$$

In this work, quasi-subordination is used to establish the coefficient estimates of a Sakaguchi type of univalent function.

\section{Main Results}

Theorem 2.1. If $f(z)$ belongs to $M_{q}^{\lambda}(\gamma, t, b)$ then

$$
\left|a_{2}\right| \leq\left|\frac{d_{0} c_{1}}{2[1]_{q} !(2-\lambda(t+b))}\right|
$$




$$
\begin{aligned}
& \left|a_{3}\right| \leq \frac{1}{3-\lambda\left(t^{2}+t b+b^{2}\right)} \mid \frac{c_{1} d_{1}}{2[1]_{q} !}+\left(\frac{c_{2}}{2[1]_{q} !}+A c_{1}^{2}\right) d_{0}-\left(\frac{\lambda(\lambda+1)(t+b)^{2}}{2 !}-2 \lambda(t+b)\right) \\
& \left(\frac{d_{0}^{2} c_{1}^{2}}{4\left([1]_{q} !\right)^{2}(2-\lambda(t+b))^{2}}\right) \mid \\
& \left|a_{4}\right| \leq \frac{1}{4-\lambda\left(t^{3}+t^{2} b+t b^{2}+b^{3}\right)} \mid \frac{c_{1} d_{2}}{2[1]_{q} !}+\left(\frac{c_{2}}{2[1]_{q} !}+A c_{1}^{2}\right) d_{1}+\left(\frac{c_{3}}{2[1]_{q} !}+2 A c_{1} c_{2}+B c_{1}^{3}\right) d_{0} \\
& -\left(\lambda(\lambda+1)(t+b)^{2}-\frac{\lambda(\lambda+1)(\lambda+2)(t+b)^{3}}{3 !}\right)\left(\frac{d_{0}^{3} c_{1}^{3}}{8\left([1]_{q} !\right)^{3}(2-\lambda(t+b))^{3}}\right) \\
& -\left(\lambda(\lambda+1)\left(t^{3}+t^{2} b+t b^{2}+b^{3}\right)-2 \lambda\left(t^{2}+t b+b^{2}\right)\right)-3 \lambda(t+b)\left(\frac{d_{0} c_{1}}{2[1]_{q} !(2-\lambda(t+b))\left(3-\lambda\left(t^{2}+t b+b^{2}\right)\right)}\right) \\
& \left(\frac{c_{1} d_{1}}{2[1]_{q} !}+\left(\frac{c_{2}}{2[1]_{q} !}+A c_{1}^{2}\right) d_{0}-\left(\frac{\lambda(\lambda+1)(t+b)^{2}}{2 !}+2 \lambda(t+b)\right)\left(\frac{d_{1}^{2} c_{1}^{2}}{4\left([1]_{q} !\right)^{2}(2-\lambda(t+b))^{2}}\right)\right) \mid
\end{aligned}
$$

where

$$
\begin{aligned}
A & =\left(\frac{[2]_{q} !-2\left([1]_{q} !\right)^{2}}{4\left([1]_{q} !\right)^{2}[2]_{q} !}\right) \\
B & =\left(\frac{4\left([1]_{q} !\right)^{3}[2]_{q} !-4\left([1]_{q} !\right)^{2}[3]_{q} !+[2]_{q} ![3]_{q} !}{8\left([1]_{q} !\right)^{3}[2]_{q} ![3]_{q} !}\right)
\end{aligned}
$$

Proof:

Suppose $f(z) \in M_{q}^{\lambda}(\gamma, t, b)$, then by definition,

$$
\frac{f^{\prime}(z)\left(\frac{(t-b) z}{f(t z)-f(b z)}\right)^{\lambda}-1}{\varphi(z)}=\gamma_{q, m, n}(z)-1,(z \in \mathbb{U})
$$

$$
\begin{gathered}
f^{\prime}(z)\left(\frac{(t-b) z}{f(t z)-f(b z)}\right)^{\lambda}-1=\varphi(z)\left(\gamma_{q, m, n}(z)-1\right) \\
f(t z)-f(b z)=(t-b) z+\left(t^{2}-b^{2}\right) a_{2} z^{2}+\left(t^{3}-b^{3}\right) a_{3} z^{3}+\left(t^{4}-b^{4}\right) a_{4} z^{4}+\ldots \\
f^{\prime}(z)=1+2 a_{2} z+3 a_{3} z^{2}+4 a_{4} z^{3}+\ldots \\
f^{\prime}(z)\left(\frac{(t-b) z}{f(t z)-f(b z)}\right)^{\lambda}= \\
f^{\prime}(z)\left[((t-b) z)\left((t-b) z+\left(t^{2}-b^{2}\right) a_{2} z^{2}+\left(t^{3}-b^{3}\right) a_{3} z^{3}+\left(t^{4}-b^{4}\right) a_{4} z^{4}+\ldots\right)^{-1}\right]^{\lambda} \\
f^{\prime}(z)\left[((t-b) z)((t-b) z)^{-1}\left(1+(t+b) a_{2} z+\left(t^{2}+t b+b^{2}\right) a_{3} z^{2}+\left(t^{3}+t^{2} b+t b^{2}+b^{3}\right) a_{4} z^{3} \ldots\right)^{-1}\right]^{\lambda} \\
f^{\prime}(z)\left(1+(t+b) a_{2} z+\left(t^{2}+t b+b^{2}\right) a_{3} z^{2}+\left(t^{3}+t^{2} b+t b^{2}+b^{3}\right) a_{4} z^{3} \ldots\right)^{-\lambda}
\end{gathered}
$$

Applying the Binomial expansion, we have,

$$
\begin{aligned}
& f^{\prime}(z)\left(\frac{(t-b) z}{f(t z)-f(b z)}\right)^{\lambda}-1= \\
& (2-\lambda(t+b)) a_{2} z+\left(\frac{(\lambda)(\lambda+1)}{2 !}(t+b)^{2} a_{2}^{2}-\lambda\left(t^{2}+t b+b^{2}\right) a_{3}-2 \lambda(t-b) a_{2}^{2}+3 a_{3}\right) z^{2} \\
& +\left(\lambda(\lambda+1)\left(t^{3}+t^{2} b+t b^{2}+b^{3}\right) a_{2} a_{3}-\lambda\left(t^{3}+t^{2} b+t b^{2}+b^{3}\right) a_{4}-\frac{(\lambda)(\lambda+1)(\lambda+2)}{3 !}(t+b)^{3} a_{2}^{3}+\right. \\
& \left.\lambda(\lambda+1)(t+b)^{2} a_{2}^{3}-2 \lambda\left(t^{2}+t b+b^{2}\right) a_{2} a_{3}-3 \lambda(t-b) a_{2} a_{3}+4 a_{4}\right) z^{3}+\ldots
\end{aligned}
$$

Furthermore,

$$
\gamma_{q, m, n}(\omega(z))=1+\frac{c_{1}}{2[1]_{q} !} z+\left(\frac{c_{2}}{2[1]_{q} !}+A c_{1}^{2}\right) z^{2}+\left(\frac{c_{3}}{2[1]_{q} !}+2 A c_{1} c_{2}+B c_{1}^{3}\right) z^{3}+\ldots
$$


Also,

$$
\varphi(z)=d_{0}+d_{1} z+d_{2} z^{2}+\ldots
$$

$$
\begin{aligned}
& \varphi(z)\left(\gamma_{q, m, n}(\omega(z))-1\right)= \\
& \left(d_{0}+d_{1} z+d_{2} z^{2}+\ldots\right)\left(\frac{c_{1}}{2[1]_{q} !} z+\left(\frac{c_{2}}{2[1]_{q} !}+A c_{1}^{2}\right) z^{2}+\left(\frac{c_{3}}{2[1]_{q} !}+2 A c_{1} c_{2}+B c_{1}^{3}\right) z^{3}+\ldots\right)
\end{aligned}
$$

then,

$$
\begin{aligned}
& d_{0}\left(\frac{c_{1}}{2[1]_{q} !} z+\left(\frac{c_{2}}{2[1]_{q} !}+A c_{1}^{2}\right) z^{2}+\left(\frac{c_{3}}{2[1]_{q} !}+2 A c_{1} c_{2}+B c_{1}^{3}\right) z^{3}+\ldots\right)+d_{1} z\left(\frac{c_{1}}{2[1]_{q} !} z+\left(\frac{c_{2}}{2[1]_{q} !}+A c_{1}^{2}\right) z^{2}+\ldots\right)+ \\
& d_{2} z^{2}\left(\frac{c_{1}}{2[1]_{q} !} z+\left(\frac{c_{2}}{2[1]_{q} !}+\ldots\right) \frac{d_{0} c_{1}}{2[1]_{q} !} z+\left(\frac{c_{2}}{2[1]_{q} !}+A c_{1}^{2}\right) d_{0} z^{2}+\left(\frac{c_{3}}{2[1]_{q} !}+2 A c_{1} c_{2}+B c_{1}^{3}\right) d_{0} z^{3}\right. \\
& +\frac{d_{1} c_{1}}{2[1]_{q} !} z^{2}+\left(\frac{c_{2}}{2[1]_{q} !}+A c_{1}^{2}\right) d_{1} z^{3}+\frac{d_{2} c_{1}}{2[1]_{q} !} z^{3} \ldots \\
& \varphi(z)\left(\gamma_{q, m, n}(\omega(z))-1\right)=\frac{d_{0} c_{1}}{2[1]_{q} !} z+\left(\frac{d_{1} c_{1}}{2[1]_{q} !}+\left(\frac{c_{2}}{2[1]_{q} !}+A c_{1}^{2}\right) d_{0}\right) z^{2}+\left(\frac{d_{2} c_{1}}{2[1]_{q} !} z^{3}+\left(\frac{c_{2}}{2[1]_{q} !}+A c_{1}^{2}\right) d_{1}\right. \\
& \left.+\left(\frac{c_{3}}{2[1]_{q} !}+2 A c_{1} c_{2}+B c_{1}^{3}\right) d_{0}\right) z^{3}+\ldots
\end{aligned}
$$

Equating (11) and (14), we have,

$(2-\lambda(t+b)) a_{2} z+\left(\frac{(\lambda)(\lambda+1)}{2 !}(t+b)^{2} a_{2}^{2}-\lambda\left(t^{2}+t b+b^{2}\right) a_{3}-2 \lambda(t-b) a_{2}^{2}+3 a_{3}\right) z^{2}$

$+\left(\lambda(\lambda+1)\left(t^{3}+t^{2} b+t b^{2}+b^{3}\right) a_{2} a_{3}-\lambda\left(t^{3}+t^{2} b+t b^{2}+b^{3}\right) a_{4}-\frac{(\lambda)(\lambda+1)(\lambda+2)}{3 !}(t+b)^{3} a_{2}^{3}+\right.$

$\left.\lambda(\lambda+1)(t+b)^{2} a_{2}^{3}-2 \lambda\left(t^{2}+t b+b^{2}\right) a_{2} a_{3}-3 \lambda(t-b) a_{2} a_{3}+4 a_{4}\right) z^{3}+\ldots$

$=\frac{d_{0} c_{1}}{2[1]_{q} !} z+\left(\frac{d_{1} c_{1}}{2[1]_{q} !}+\left(\frac{c_{2}}{2[1]_{q} !}+A c_{1}^{2}\right) d_{0}\right) z^{2}+\left(\frac{d_{2} c_{1}}{2[1]_{q} !}+\left(\frac{c_{2}}{2[1]_{q} !}+A c_{1}^{2}\right) d_{1}+\left(\frac{c_{3}}{2[1]_{q} !}+2 A c_{1} c_{2}+B c_{1}^{3}\right) d_{0}\right) z^{3}+\ldots$

Comparing coefficients of $z, z^{2}, z^{3}$ in (15) to obtain $a_{2}, a_{3}, a_{4}$, we have

$$
\begin{gathered}
(2-\lambda(t+b)) a_{2} z=\frac{d_{0} c_{1}}{2[1]_{q} !} z \\
a_{2}=\frac{1}{2-\lambda(t+b)}\left(\frac{d_{0} c_{1}}{2[1]_{q} !}\right) \\
a_{2}=\frac{d_{0} c_{1}}{2[1]_{q} !(2-\lambda(t+b))} \\
\left|a_{2}\right| \leq\left|\frac{d_{0} c_{1}}{2[1]_{q} !(2-\lambda(t+b))}\right|
\end{gathered}
$$

Also,

$$
\begin{aligned}
& \left(\frac{(\lambda)(\lambda+1)}{2 !}(t+b)^{2} a_{2}^{2}-\lambda\left(t^{2}+t b+b^{2}\right) a_{3}-2 \lambda(t-b) a_{2}^{2}+3 a_{3}\right) z^{2}=\left(\frac{d_{1} c_{1}}{2[1]_{q} !}+\left(\frac{c_{2}}{2[1]_{q} !}+A c_{1}^{2}\right) d_{0}\right) z^{2} \\
& a_{3}=\frac{1}{\left(3-\lambda\left(t^{2}+t b+b^{2}\right)\right)}\left(\frac{d_{1} c_{1}}{2[1]_{q} !}+\left(\frac{c_{2}}{2[1]_{q} !}+A c_{1}^{2}\right) d_{0}-\left(\frac{(\lambda)(\lambda+1)}{2 !}(t+b)^{2}-2 \lambda(t-b)\right)\left(\frac{d_{0} c_{1}}{2[1]_{q} !(2-\lambda(t+b))}\right)^{2}\right) \\
& \left|a_{3}\right| \leq \frac{1}{3-\lambda\left(t^{2}+t b+b^{2}\right)} \mid \frac{c_{1} d_{1}}{2[1]_{q} !}+\left(\frac{c_{2}}{2[1]_{q} !}+A c_{1}^{2}\right) d_{0}-\left(\frac{\lambda(\lambda+1)(t+b)^{2}}{2 !}-2 \lambda(t+b)\right) \\
& \left(\frac{d_{0}^{2} c_{1}^{2}}{4\left([1]_{q} !\right)^{2}(2-\lambda(t+b))^{2}}\right) \mid
\end{aligned}
$$




\section{Furthermore,}

$$
\begin{aligned}
& \left(\lambda(\lambda+1)\left(t^{3}+t^{2} b+t b^{2}+b^{3}\right) a_{2} a_{3}-\lambda\left(t^{3}+t^{2} b+t b^{2}+b^{3}\right) a_{4}-\frac{(\lambda)(\lambda+1)(\lambda+2)}{3 !}(t+b)^{3} a_{2}^{3}+\lambda(\lambda+1)(t+b)^{2} a_{2}^{3}-\right. \\
& \left.2 \lambda\left(t^{2}+t b+b^{2}\right) a_{2} a_{3}-3 \lambda(t-b) a_{2} a_{3}+4 a_{4}\right) z^{3}+\ldots=\left(\frac{d_{2} c_{1}}{2[1]_{q} !}+\left(\frac{c_{2}}{2[1]_{q} !}+A c_{1}^{2}\right) d_{1}+\left(\frac{c_{3}}{2[1]_{q} !}+2 A c_{1} c_{2}+B c_{1}^{3}\right) d_{0}\right) z^{3}+\ldots \\
& a_{4}=\frac{1}{4-\lambda\left(t^{3}+t^{2} b+t b^{2}+b^{3}\right)}\left(\frac{c_{1} d_{2}}{2[1]_{q} !}+\left(\frac{c_{2}}{2[1]_{q} !}+A c_{1}^{2}\right) d_{1}+\left(\frac{c_{3}}{2[1]_{q} !}+2 A c_{1} c_{2}+B c_{1}^{3}\right) d_{0}-\right. \\
& \left(\lambda(\lambda+1)(t+b)^{2}-\frac{\lambda(\lambda+1)(\lambda+2)(t+b)^{3}}{3 !}\right)\left(\frac{d_{0}^{3} c_{1}^{3}}{8\left([1]_{q} !\right)^{3}(2-\lambda(t+b))^{3}}\right)-\left(\lambda(\lambda+1)\left(t^{3}+t^{2} b+t b^{2}+b^{3}\right)-2 \lambda\left(t^{2}+t b+b^{2}\right)\right) \\
& -3 \lambda(t+b)\left(\frac{c_{1} d_{1}}{2[1]_{q} !(2-\lambda(t+b))\left(3-\lambda\left(t^{2}+t b+b^{2}\right)\right)}\right)\left(\frac{c_{2}}{2[1]_{q} !}+\left(\frac{d_{0}^{2} c_{1}^{2}}{2[1]_{q} !}+A c_{1}^{2}\right) d_{0}-\right. \\
& \left.\left.\left(\frac{\lambda(\lambda+1)(t+b)^{2}}{2 !}+2 \lambda(t+b)\right)\left(\frac{1}{4\left([1]_{q} !\right)^{2}(2-\lambda(t+b))^{2}}\right)\right)\right) \\
& \left|a_{4}\right| \leq \frac{c_{2}}{4-\lambda\left(t^{3}+t^{2} b+t b^{2}+b^{3}\right)} \mid \frac{c_{1} d_{2}}{2[1]_{q} !}+\left(\frac{c_{2}}{2[1]_{q} !}+A c_{1}^{2}\right) d_{1}+\left(\frac{c_{3}}{2[1]_{q} !}+2 A c_{1} c_{2}+B c_{1}^{3}\right) d_{0}-\left(\lambda(\lambda+1)(t+b)^{2}-\right. \\
& \left.\frac{\lambda(\lambda+1)(\lambda+2)(t+b)^{3}}{3 !}\right)\left(\frac{d_{0}^{3} c_{1}^{3}}{8\left([1]_{q} !\right)^{3}(2-\lambda(t+b))^{3}}\right)-\left(\lambda(\lambda+1)\left(t^{3}+t^{2} b+t b^{2}+b^{3}\right)-2 \lambda\left(t^{2}+t b+b^{2}\right)\right)-3 \lambda(t+b) \\
& \left(\frac{d_{0} c_{1}}{2[1]_{q} !(2-\lambda(t+b))\left(3-\lambda\left(t^{2}+t b+b^{2}\right)\right)}\right)\left(\frac{c_{1} d_{1}}{2[1]_{q} !}+\left(\frac{c_{2}}{2[1]_{q} !}+A c_{1}^{2}\right) d_{0}-\left(\frac{\lambda(\lambda+1)(t+b)^{2}}{2 !}+2 \lambda(t+b)\right)\right. \\
& \left.\left(\frac{d_{0}^{2} c_{1}^{2}}{4\left([1]_{q} !\right)^{2}(2-\lambda(t+b))^{2}}\right)\right) \mid
\end{aligned}
$$

Remark 2.1. Setting $q=1,\left|a_{2}\right|$ and $\left|a_{3}\right|$ agree with Olatunji et.al [7]

Theorem 2.2. If $f(z)$ belongs to $M_{q}^{\lambda}(\gamma, t, b)$ and $\mu \in \mathbb{R}$ then

$$
\begin{aligned}
& \left|a_{3}-\mu a_{2}^{2}\right| \leq \frac{1}{3-\lambda\left(t^{2}+t b+b^{2}\right)} \mid \frac{c_{1} d_{1}}{2[1]_{q} !}+\left(\frac{c_{2}}{2[1]_{q} !}+A c_{1}^{2}\right) d_{0}-\left(\frac{\lambda(\lambda+1)(t+b)^{2}}{2 !}-2 \lambda(t+b)\right) \\
& \left(\frac{d_{0}^{2} c_{1}^{2}}{4\left([1]_{q} !\right)^{2}(2-\lambda(t+b))^{2}}\right)-\mu\left(\frac{d_{0}^{2} c_{1}^{2}\left(3-\lambda\left(t^{2}+t b+b^{2}\right)\right)}{4\left([1]_{q} !\right)^{2}(2-\lambda(t+b))^{2}}\right) \mid
\end{aligned}
$$

\section{Proof:}

From (16) and (17), we have,

$$
\begin{aligned}
& a_{3}-\mu a_{2}^{2}=\frac{1}{3-\lambda\left(t^{2}+t b+b^{2}\right)}\left(\frac{c_{1} d_{1}}{2[1]_{q} !}+\left(\frac{c_{2}}{2[1]_{q} !}+A c_{1}^{2}\right) d_{0}-\left(\frac{\lambda(\lambda+1)(t+b)^{2}}{2 !}-2 \lambda(t+b)\right)\right. \\
& \left.\left(\frac{d_{0}^{2} c_{1}^{2}}{4\left([1]_{q} !\right)^{2}(2-\lambda(t+b))^{2}}\right)\right)-\mu\left(\frac{1}{4\left([1]_{q} !\right)^{2}(2-\lambda(t+b))^{2}}\right) \\
& a_{3}-\mu a_{2}^{2}=\frac{1}{3-\lambda\left(t^{2}+t b+b^{2}\right)}\left(\frac{c_{1} d_{1}}{2[1]_{q} !}+\left(\frac{c_{2}}{2[1]_{q} !}+A c_{1}^{2}\right) d_{0}-\left(\frac{\lambda(\lambda+1)(t+b)^{2}}{2 !}-2 \lambda(t+b)\right)\right. \\
& \left.\left(\frac{d_{0}^{2} c_{1}^{2}}{4\left([1]_{q} !\right)^{2}(2-\lambda(t+b))^{2}}\right)-\mu\left(\frac{d_{0}^{2} c_{1}^{2}\left(3-\lambda\left(t^{2}+t b+b^{2}\right)\right)}{4\left([1]_{q} !\right)^{2}(2-\lambda(t+b))^{2}}\right)\right) \\
& \left|a_{3}-\mu a_{2}^{2}\right| \leq \frac{1}{3-\lambda\left(t^{2}+t b+b^{2}\right)} \mid \frac{c_{1} d_{1}}{2[1]_{q} !}+\left(\frac{c_{2}}{2[1]_{q} !}+A c_{1}^{2}\right) d_{0}-\left(\frac{\lambda(\lambda+1)(t+b)^{2}}{2 !}-2 \lambda(t+b)\right) \\
& \left(\frac{d_{0}^{2} c_{1}^{2}}{4\left([1]_{q} !\right)^{2}(2-\lambda(t+b))^{2}}\right)-\mu\left(\frac{d_{0}^{2} c_{1}^{2}\left(3-\lambda\left(t^{2}+t b+b^{2}\right)\right)}{4\left([1]_{q} !\right)^{2}(2-\lambda(t+b))^{2}}\right) \mid
\end{aligned}
$$

which completes the proof for the Fekete-Szegö Inequality. 
Theorem 2.3. If $f(z)$ belongs to $M_{q}^{\lambda}(\gamma, t, b)$ and $\mu \in \mathbb{R}$ then

$$
\begin{aligned}
& \left|a_{2} a_{4}-\mu a_{3}^{2}\right| \leq \mid\left(\frac { d _ { 0 } c _ { 1 } } { 2 [ 1 ] _ { q } ! ( 2 - \lambda ( t + b ) ) ( 4 - \lambda ( t ^ { 3 } + t ^ { 2 } b + t b ^ { 2 } + b ^ { 3 } ) ) } \left(\frac{c_{1} d_{2}}{2[1]_{q} !}+\left(\frac{c_{2}}{2[1]_{q} !}+A c_{1}^{2}\right) d_{1}\right.\right. \\
& +\left(\frac{c_{3}}{2[1]_{q} !}+2 A c_{1} c_{2}+B c_{1}^{3}\right) d_{0}-\left(\lambda(\lambda+1)(t+b)^{2}-\frac{\lambda(\lambda+1)(\lambda+2)(t+b)^{3}}{3 !}\right)\left(\frac{d_{0}^{3} c_{1}^{3}}{8\left([1]_{q} !\right)^{3}(2-\lambda(t+b))^{3}}\right)- \\
& \left(\lambda(\lambda+1)\left(t^{3}+t^{2} b+t b^{2}+b^{3}\right)-2 \lambda\left(t^{2}+t b+b^{2}\right)\right)-3 \lambda(t+b)\left(\frac{d_{0} c_{1}}{2[1]_{q} !(2-\lambda(t+b))\left(3-\lambda\left(t^{2}+t b+b^{2}\right)\right)}\right)\left(\frac{c_{1} d_{1}}{2[1]_{q} !}\right. \\
& \left.\left.+\left(\frac{c_{2}}{2[1]_{q} !}+A c_{1}^{2}\right) d_{0}-\left(\frac{\lambda(\lambda+1)(t+b)^{2}}{2 !}+2 \lambda(t+b)\right)\left(\frac{1}{4\left([1]_{q} !\right)^{2}(2-\lambda(t+b))^{2}}\right)\right)\right)-\frac{\mu}{3-\lambda\left(t^{2}+t b+b^{2}\right)^{2}} \\
& \left(\frac{d_{1}^{2} c_{1}^{2}}{4\left([1]_{q} !\right)^{2}}+\left(\frac{c_{2}}{2[1]_{q} !}+A c_{1}^{2}\right)^{2} d_{0}^{2}+\left(\frac{(\lambda)(\lambda+1)}{2 !}(t+b)^{2}-2 \lambda(t-b)\right)^{2}\left(\frac{d_{0} c_{1}}{2[1]_{q} !(2-\lambda(t+b))}\right)^{4}+\left(\frac{c_{1} c_{2} d_{1}}{2[1]_{q} !}+\right.\right. \\
& \left.\frac{A c_{1}^{3} d_{1}}{[1]_{q} !}\right)_{0}-\left(\frac{(\lambda)(\lambda+1)}{2 !}(t+b)^{2}-2 \lambda(t-b)\right)\left(\frac{(\lambda)(\lambda+1)}{4\left([1]_{q} !\right)^{3}(2-\lambda(t+b))^{2}}\right)-\left(\frac{d_{0}^{2} c_{1}^{3} d_{1}}{2 !}(t+b)^{2}-2 \lambda(t-b)\right) \\
& \left(\frac{d_{0}^{2} c_{1}^{2} c_{2}}{4\left([1]_{q} !\right)^{3}(2-\lambda(t+b))^{2}}+\frac{A c_{1}^{4} d_{0}}{2\left([1]_{q} !\right)^{2}(2-\lambda(t+b))^{2}}\right) \mid
\end{aligned}
$$

\section{Proof:}

From (16), (17) and (18), we have,

$$
\begin{aligned}
& a_{2} a_{4}-\mu a_{3}^{2}=\left(\frac{d_{0} c_{1}}{2[1]_{q} !(2-\lambda(t+b))}\right)\left(\frac { 1 } { 4 - \lambda ( t ^ { 3 } + t ^ { 2 } b + t b ^ { 2 } + b ^ { 3 } ) } \left(\frac{c_{1} d_{2}}{2[1]_{q} !}+\left(\frac{c_{2}}{2[1]_{q} !}+A c_{1}^{2}\right) d_{1}\right.\right. \\
& +\left(\frac{c_{3}}{2[1]_{q} !}+2 A c_{1} c_{2}+B c_{1}^{3}\right) d_{0}-\left(\lambda(\lambda+1)(t+b)^{2}-\frac{\lambda(\lambda+1)(\lambda+2)(t+b)^{3}}{3 !}\right)\left(\frac{d_{0}^{3} c_{1}^{3}}{8\left([1]_{q} !\right)^{3}(2-\lambda(t+b))^{3}}\right)- \\
& \left(\lambda(\lambda+1)\left(t^{3}+t^{2} b+t b^{2}+b^{3}\right)-2 \lambda\left(t^{2}+t b+b^{2}\right)\right)-3 \lambda(t+b)\left(\frac{d_{0} c_{1}}{2[1]_{q} !(2-\lambda(t+b))\left(3-\lambda\left(t^{2}+t b+b^{2}\right)\right)}\right)\left(\frac{c_{1} d_{1}}{2[1]_{q} !}+\right. \\
& \left.\left.\left(\frac{c_{2}}{2[1]_{q} !}+A c_{1}^{2}\right) d_{0}-\left(\frac{\lambda(\lambda+1)(t+b)^{2}}{2 !}+2 \lambda(t+b)\right)\left(\frac{d_{0}^{2} c_{1}^{2}}{4\left([1]_{q} !\right)^{2}(2-\lambda(t+b))^{2}}\right)\right)\right)-\mu\left(\frac{1}{\left(3-\lambda\left(t^{2}+t b+b^{2}\right)\right)}\right. \\
& \left.\left(\frac{d_{1} c_{1}}{2[1]_{q} !}+\left(\frac{c_{2}}{2[1]_{q} !}+A c_{1}^{2}\right) d_{0}-\left(\frac{(\lambda)(\lambda+1)}{2 !}(t+b)^{2}-2 \lambda(t-b)\right)\left(\frac{d_{0} c_{1}}{2[1]_{q} !(2-\lambda(t+b))}\right)^{2}\right)\right)^{2} \\
& a_{2} a_{4}-\mu a_{3}^{2}=\left(\frac{c_{0} c_{1}}{2[1]_{q} !(2-\lambda(t+b))}\right)\left(\frac { c _ { 1 } } { 4 - \lambda ( t ^ { 3 } + t ^ { 2 } b + t b ^ { 2 } + b ^ { 3 } ) } \left(\frac{c_{2}[1]_{q} !}{2}+\left(\frac{c_{2}}{2[1]_{q} !}+A c_{1}^{2}\right) d_{1}\right.\right. \\
& +\left(\frac{c_{3}}{2[1]_{q} !}+2 A c_{1} c_{2}+B c_{1}^{3}\right) d_{0}-\left(\lambda(\lambda+1)(t+b)^{2}-\frac{\lambda(\lambda+1)(\lambda+2)(t+b)^{3}}{3 !\left([1]_{q} !\right)^{3}(2-\lambda(t+b))^{3}}\right)- \\
& \left(\lambda(\lambda+1)\left(t^{3}+t^{2} b+t b^{2}+b^{3}\right)-2 \lambda\left(t^{2}+t b+b^{2}\right)\right)-3 \lambda(t+b)\left(\frac{d_{0} c_{1}}{2[1]_{q} !(2-\lambda(t+b))\left(3-\lambda\left(t^{2}+t b+b^{2}\right)\right)}\right)\left(\frac{c_{1} d_{1}}{2[1]_{q} !}+\right. \\
& \left.\left.\left.\left(\frac{c_{2}}{2[1]_{q} !}+A c_{1}^{2}\right) d_{0}-\left(\frac{\lambda(\lambda+1)(t+b)^{2}}{2 !}+2 \lambda(t+b)\right)\left(\frac{d_{0}^{2} c_{1}^{2}}{4\left([1]_{q} !\right)^{2}(2-\lambda(t+b))^{2}}\right)\right)\right)\right)-\frac{\mu}{3-\lambda\left(t^{2}+t b+b^{2}\right)^{2}} \\
& \left(\frac{d_{1}^{2} c_{1}^{2}}{4\left([1]_{q} !\right)^{2}}+\left(\frac{c_{2}}{2[1]_{q} !}+A c_{1}^{2}\right)^{2} d_{0}^{2}+\left(\frac{(\lambda)(\lambda+1)}{2 !}(t+b)^{2}-2 \lambda(t-b)\right)^{2}\left(\frac{d_{0} c_{1}}{2[1]_{q} !(2-\lambda(t+b))}\right)+\left(\frac{c_{1} c_{2} d_{1}}{2[1]_{q} !}+\right.\right. \\
& \left.\frac{A c_{1}^{3} d_{1}}{[1]_{q} !}\right) d_{0}-\left(\frac{(\lambda)(\lambda+1)}{2 !}(t+b)^{2}-2 \lambda(t-b)\right)\left(\frac{d_{0}^{2} c_{1}^{3} d_{1}}{4\left([1]_{q} !\right)^{3}(2-\lambda(t+b))^{2}}\right)-\left(\frac{1 \lambda+1)}{2 !}(t+b)^{2}-2 \lambda(t-b)\right) \\
& \left(\frac{d_{0}^{2} c_{1}^{2} c_{2}}{4\left([1]_{q} !\right)^{3}(2-\lambda(t+b))^{2}}+\frac{A c_{1}^{4} d_{0}}{2\left([1]_{q} !\right)^{2}(2-\lambda(t+b))^{2}}\right)
\end{aligned}
$$

$$
\begin{aligned}
& \left|a_{2} a_{4}-\mu a_{3}^{2}\right| \leq \mid\left(\frac { d _ { 0 } c _ { 1 } } { 2 [ 1 ] _ { q } ! ( 2 - \lambda ( t + b ) ) ( 4 - \lambda ( t ^ { 3 } + t ^ { 2 } b + t b ^ { 2 } + b ^ { 3 } ) ) } \left(\frac{c_{1} d_{2}}{2[1]_{q} !}+\left(\frac{c_{2}}{2[1]_{q} !}+A c_{1}^{2}\right) d_{1}\right.\right. \\
& +\left(\frac{c_{3}}{2[1]_{q} !}+2 A c_{1} c_{2}+B c_{1}^{3}\right) d_{0}-\left(\lambda(\lambda+1)(t+b)^{2}-\frac{\lambda(\lambda+1)(\lambda+2)(t+b)^{3}}{3 !}\right)\left(\frac{d_{0}^{3} c_{1}^{3}}{8\left([1]_{q} !\right)^{3}(2-\lambda(t+b))^{3}}\right)- \\
& \left(\lambda(\lambda+1)\left(t^{3}+t^{2} b+t b^{2}+b^{3}\right)-2 \lambda\left(t^{2}+t b+b^{2}\right)\right)-3 \lambda(t+b)\left(\frac{d_{0} c_{1}}{2[1]_{q} !(2-\lambda(t+b))\left(3-\lambda\left(t^{2}+t b+b^{2}\right)\right)}\right)\left(\frac{c_{1} d_{1}}{2[1]_{q} !}\right. \\
& \left.\left.+\left(\frac{c_{2}}{2[1]_{q} !}+A c_{1}^{2}\right) d_{0}-\left(\frac{\lambda(\lambda+1)(t+b)^{2}}{2 !}+2 \lambda(t+b)\right)\left(\frac{d_{0}^{2} c_{1}^{2}}{4\left([1]_{q} !\right)^{2}(2-\lambda(t+b))^{2}}\right)\right)\right)-\frac{\mu}{3-\lambda\left(t^{2}+t b+b^{2}\right)^{2}} \\
& \left(\frac{d_{1}^{2} c_{1}^{2}}{4\left([1]_{q} !\right)^{2}}+\left(\frac{c_{2}}{2[1]_{q} !}+A c_{1}^{2}\right)^{2} d_{0}^{2}+\left(\frac{(\lambda)(\lambda+1)}{2 !}(t+b)^{2}-2 \lambda(t-b)\right)^{2}\left(\frac{d_{0} c_{1}}{2[1]_{q} !(2-\lambda(t+b))}\right)^{4}+\left(\frac{c_{1} c_{2} d_{1}}{2[1]_{q} !}+\right.\right. \\
& \left.\frac{A c_{1}^{3} d_{1}}{[1]_{q} !}\right) d_{0}-\left(\frac{(\lambda)(\lambda+1)}{2 !}(t+b)^{2}-2 \lambda(t-b)\right)\left(\frac{(\lambda)(\lambda+1)}{4\left([1]_{q} !\right)^{3}(2-\lambda(t+b))^{2}}\right)-\left(\frac{d_{0}^{2} c_{1}^{3} d_{1}}{2 !}(t+b)^{2}-2 \lambda(t-b)\right) \\
& \left(\frac{d_{0}^{2} c_{1}^{2} c_{2}}{4\left([1]_{q} !\right)^{3}(2-\lambda(t+b))^{2}}+\frac{A c_{1}^{4} d_{0}}{2\left([1]_{q} !\right)^{2}(2-\lambda(t+b))^{2}}\right)
\end{aligned}
$$

which completes the proof for Hankel Determinant. 


\section{REFERENCES}

[1] $\ddot{O}$. Afet, $q$-Polynomials and location of their zero, PhD Thesis, Eastern Mediterranean University Gazimağusa, North Cyprus, (2014).

[2] P. L. Duren, Univalent Functions. Springer-Verlag, New York Incoporation, (1983).

[3] J. Dziok, A general solution of the Fekete-Szegö problem, Bound. Value Probl. 2013 (2013), 98. https: //doi.org/10.1186/1687-2770-2013-98.

[4] U.A. Ezeafuluke, M. Darus, O.A. Fadipe-Joseph, The $q$-analogue of sigmoid function in the space of univalent $\lambda$-Pseudo star-like functions, Int. J. Math. Computer Sci. 15 (2020), 621-626.

[5] O.A. Fadipe-Joseph, A.T. Oladipo, A.U. Ezeafulukwe, Modified sigmoid function in univalent function theory, Int. J. Math. Sci. Eng. Appl. 7 (2013), 313-317.

[6] B.A. Frasin, Coefficient inequality for certain classes of Sakaguchi type functions, Int. J. Nonlinear Sci. 10 (2010), 206-211.

[7] S. O. Olatunji, On a Sakaguchi type class of analytic functions associated with Quasi-subordination in the space of modified sigmoid functions, Electron. J. Math. Anal. Appl. 5 (2017), 97-105. 